\title{
Takotsubo cardiomyopathy complicated by cardiac tamponade due to non- hemorrhagic pericardial effusion: a case report
}

\author{
Yuta Nagamori, Takuto Hamaoka* ${ }^{*}$, Hisayoshi Murai, Shinichiro Takashima, Takeshi Kato, Soichiro Usui, \\ Kenji Sakata, Hiroshi Furusho, Masaaki Kawashiri and Masayuki Takamura
}

\begin{abstract}
Background: Cardiac tamponade is a rare but serious complication of Takotsubo cardiomyopathy (TC). Two cases of cardiac tamponade subsequent to TC have been reported. The pericardial effusion in these cases was hemorrhagic and caused by ventricular rupture. Cardiac tamponade induced by an inflammatory effusion complicated with TC has not been reported. This is the first case report of TC, which developed cardiac tamponade during the recovery phase with a large volume non-hemorrhagic inflammatory effusion.

Case presentation: We describe a case of an 81 -year-old woman admitted to our hospital because of severe chest pain. Her symptoms began soon after her son's hospitalization. We diagnosed her with TC based on results of an electrocardiogram, echocardiogram, and emergent coronary angiography. Her symptoms and left ventricular dysfunction improved gradually. She developed newly confirmed chest pain and dyspnea on day 9 after admission. A large pericardial effusion developed, resulting in cardiac tamponade. Her symptoms and hemodynamic status improved immediately after the pericardiocentesis. The effusion was non-hemorrhagic and exudative. No specific signs of infection, collagen disease, or malignant tumors were observed, except for TC.

Conclusions: We experienced a case of circulatory collapse induced by TC-related inflammatory pericardial effusion at recovery phase. This case emphasizes the importance of careful follow-up even after improved left ventricular dysfunction in a patient with TC.
\end{abstract}

Keywords: Takotsubo cardiomyopathy, Pericarditis, Cardiac tamponade

\section{Background}

Takotsubo cardiomyopathy (TC) is characterized by transient dysfunction of the left ventricular mid-apical segments without significant coronary artery stenosis or occlusion that is generally induced by emotional or physical stress. TC always has a benign course; however, serious complications can develop, including cardiogenic shock, dysrhythmia, or ventricular rupture. Pericardial effusion is another well-known complication of TC, but it rarely affects the hemodynamic status. Two cases of cardiac tamponade subsequent to $\mathrm{TC}$ have been

\footnotetext{
* Correspondence: tokuta114@yahoo.co.jp

Department of Cardiology, Graduate School of Medical Science, Kanazawa University, 13-1 Takara-machi, Kanazawa 920-8641, Japan
}

reported [1, 2]. The pericardial effusion in these cases was hemorrhagic and caused by ventricular rupture. Cardiac tamponade induced by an inflammatory effusion complicated with TC has not been reported. Here, we report a patient with TC who developed cardiac tamponade during the recovery phase with a large volume non-hemorrhagic inflammatory effusion.

\section{Case presentation}

An 81-year-old woman presented to our hospital with severe chest pain for the prior 3 days. She had no medical history. She had never smoked and had no family history of cardiovascular disease. The symptoms began soon after her son was admitted to the hospital. 
a)

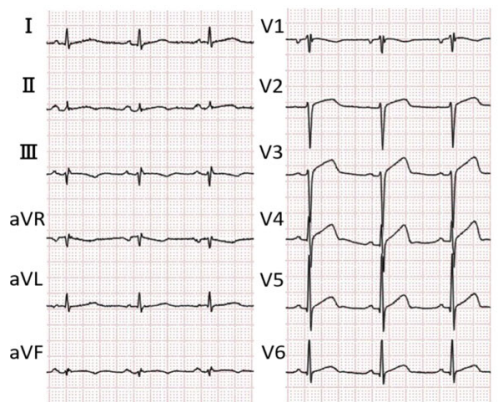

b)

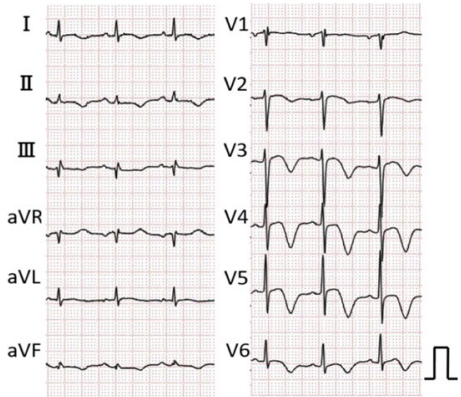

Fig. 1 ECG (a: at presentation b: on day 9). a ST-segment was elevated in leads V2 to V5, III, and aVF. b T waves were inverted in the leads where the ST segment had been elevated previously

The initial electrocardiogram (ECG) revealed a normal sinus rhythm with ST-segment elevation in leads V2 to V5, III, and aVF (Fig. 1-a). A chest X-ray showed no pulmonary congestion or pleural effusion. Echocardiography revealed akinesis in the left ventricular apical region with hypercontraction in the basal region. No pericardial effusion was observed (Fig. 2). Laboratory studies demonstrated a small elevation in cardiac enzymes: creatine kinase, $125 \mathrm{IU} / \mathrm{l}$ (normal, 32-170 IU/l); creatine kinase-MB isoenzyme, $25 \mathrm{IU} / \mathrm{l}(<16 \mathrm{IU} / \mathrm{l})$; troponin $\mathrm{T}, 0.026 \mathrm{ng} / \mathrm{mL}$. Neither neutrophils nor C-reactive protein were elevated $(\sim$ $0.03 \mathrm{mg} / \mathrm{dl}$ ). Coronary angiography was performed, but no significant coronary artery stenosis or occlusion was detected (Fig. 3). Metabolic blood flow mismatches were detected in the left ventricular apical region on a nuclear cardiology examination (Fig. 4); these were inconsistent with the coronary artery perfusion area. From these results, this patient was diagnosed with Takotsubo cardiomyopathy (TC). Her symptoms had completely improved by 3 days after admission, and cardiac rehabilitation with a careful follow-up proceeded.

On day 9 of hospitalization, she felt mild chest pain at rest, and severe exertional dyspnea. An ECG revealed inverted T-waves in the leads where the ST segment had been elevated previously (Fig. 1-b). The left ventricular dysfunction had improved on echocardiography, but pericardial effusion of up to $10 \mathrm{~mm}$ appeared behind the left ventricle inferior-lateral region, indicating pericarditis. Loxoprofen $75 \mathrm{mg}$ and colchicine $0.5 \mathrm{mg}$ per day were administered, but the pericardial effusion increased gradually, leading to collapse of the right ventricle with sinus tachycardia (Fig. 5). A paradoxical pulse was also detected, with systolic blood pressure dropping to 21 $\mathrm{mmHg}$ during the inspiration phase. Cardiac catheterization revealed equilibration of the average intracardiac diastolic pressure between the left and right ventricles, and $>20 \mathrm{mmHg}$ systolic blood pressure reduction by inspiration; these results were consistent with a)

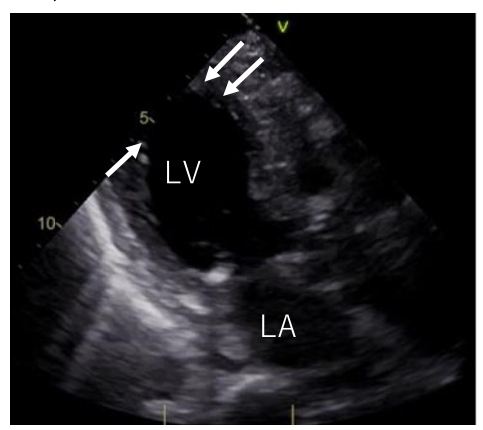

b)

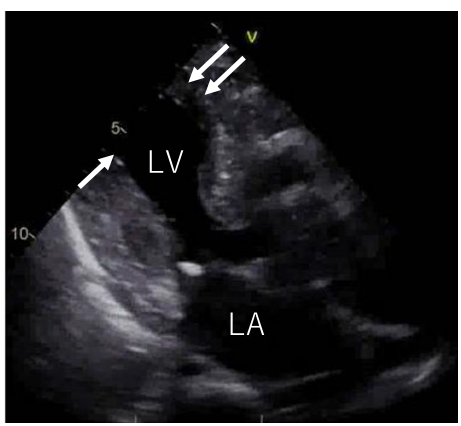

Fig. 2 Echocardiography at presentation (a: diastole b: systole). The apical region of the left ventricle was akinetic (white arrows), and there was no pericardial effusion.(LA, Left Artium; LV, Left Ventricle) 
a)

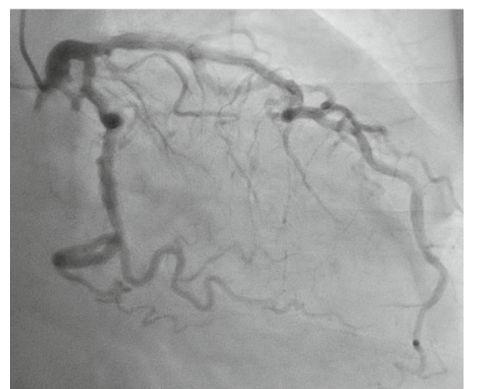

b)

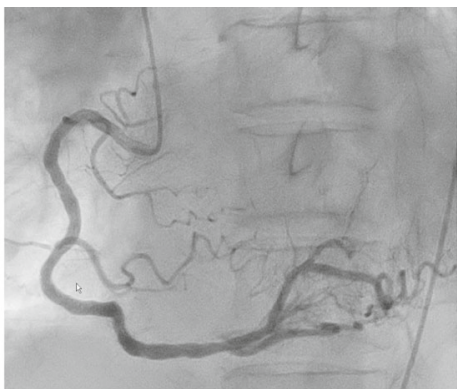

Fig. 3 Coronary angiography. a Right anterior oblique $30^{\circ} \mathbf{b}$ Left anterior oblique $45^{\circ}$. No significant coronary artery stenosis or occlusion was detected

cardiac tamponade (Fig. 6). Pericardiocentesis was performed on day 23. The pericardial effusion was nonhemorrhagic and exudative. Malignant tumors, collagen disease, and tuberculosis were not thought to have introduced the pericardial effusion (WBC 0.45, TP 5.0 IU-L, LDH $291 \mathrm{IU} / \mathrm{L}$, ADA $9.0 \mathrm{U} / \mathrm{L}$, CEA 0.3, SCC 0.6, NSE $2.7 \mathrm{ng} / \mathrm{mL}$, CYFRA 10.8, antinuclear antibody 80, cytodiagnosis negative). The serum virus paired antibody test was negative for echovirus and coxsackie virus. Therefore, only TC was a suspected cause of the pericarditis. On day 30, cardiac magnetic resonance imaging (CMRI) was performed to evaluate myocardial involvement, and no delayed gadolinium enhancement (DGE) was observed (Fig. 7). After draining $480 \mathrm{ml}$ of fluid, the patient's symptoms and abnormal hemodynamic status improved immediately. No additional pericardial effusion or relapse of symptoms has been observed.

\section{Discussion and conclusions}

The present case exemplifies two important clinical issues. First, TC can induce pericarditis even during the recovery phase. Second, TC-related inflammatory effusion is capable of disrupting hemodynamic status.

\section{Relationship between Takotsubo cardiomyopathy and pericarditis}

Some cases of pericarditis complicated with TC have been reported; however, the mechanism remains unclear. Two hypotheses for the relationship have been suggested. One is that pericarditis precedes the onset of $\mathrm{TC}$, and intense sympathetic stimulation due to severe pericarditis-related chest discomfort subsequently induces the TC [3]. The other hypothesis is that myocardial inflammation of TC spreads to the pericardium, resulting in pericarditis [4]. In the present case, echocardiography initially demonstrated left ventricular dysfunction without pericardial effusion, and the pericardial effusion developed after amelioration of left ventricular function. The results of coronary angiography, a nuclear cardiology examination, and anamnesis indicated that the left ventricular dysfunction in the present case was derived from TC. The effusion sample was exudative; however, no remarkable signs suggesting malignant a)
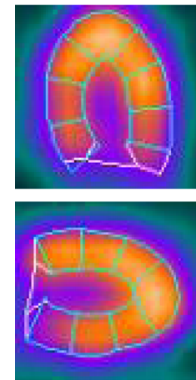

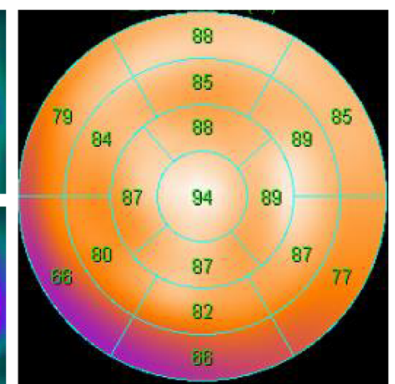

b)
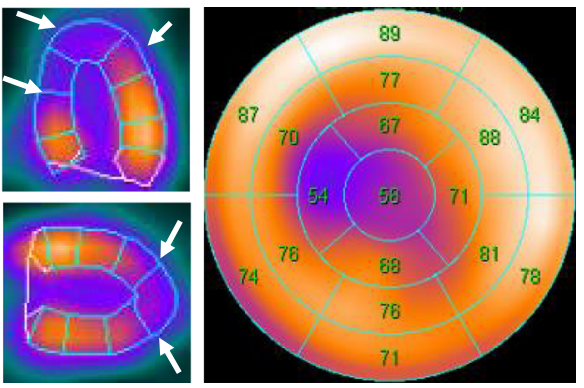

Fig. 4 Nuclear cardiology examination (a: ${ }^{201}$ Thallium b: ${ }^{123}$ - $\beta$-methyl-p-iodophenyl-pentadecanoic acid). Metabolic blood flow mismatches were detected in the left ventricular apical region (white arrows) 


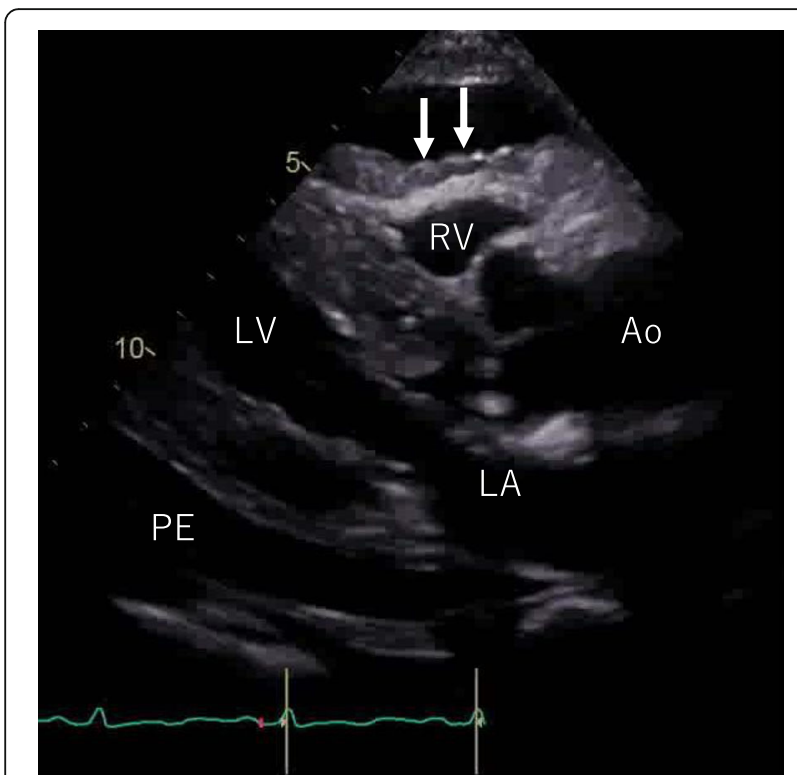

Fig. 5 Echocardiography on day 9 (diastole). Pericardial effusion up to $10 \mathrm{~mm}$ appeared behind the left ventricle inferior-lateral lesion, leading to the collapse of the right ventricle (white arrows) (LA, Left Atrium; LV, Left Ventricle; RV, Right Ventricle; Ao, Aorta; PE, Pericardial Effusion)

diseases, infection, or a collagen disease were observed. We speculate that the TC developed first, and the myocardium inflammation from TC induced pericarditis, which supports the hypothesis that $\mathrm{TC}$ precedes pericarditis. It can be difficult to differentiate TC from a coronary artery multi-vessel spasm or myocarditis [5]. In the present case, the cardiac enzymes were too low to be indicative of ischemic heart disease involving multiple vessels. In addition, CMRI demonstrated no region of DGE. A previous study showed that typical DGE is usually observed in ischemic heart disease and myocarditis;

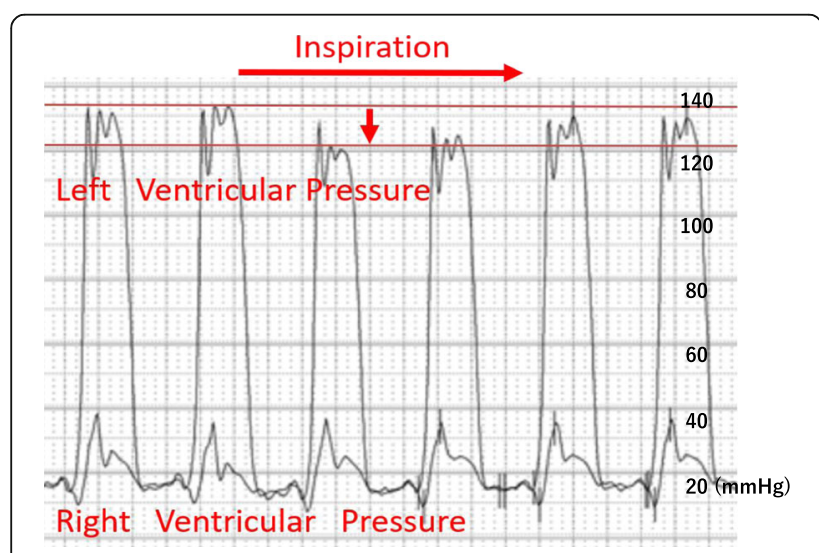

Fig. 6 Cardiac catheterization. Average intracardiac diastolic pressures was equilibrated, and systolic blood pressure was reduced more than $20 \mathrm{mmHg}$ by inspiration however, it is not observed in $95 \%$ of patients with TC [6]. These results confirmed our diagnosis of TC and TC-related pericarditis.

\section{Severity of pericarditis complicated with TC}

Pericardial effusion complicated with $\mathrm{TC}$ is not rare. Ingo et al. reported that $43 \%$ of patients with $\mathrm{TC}$ develop pericardial effusion, and $81 \%$ of patients with TC have demonstrated myocardial edema on CMRI [7], but most of these cases were asymptomatic. Some symptomatic pericarditis cases complicated with TC have been reported [8]. In most of these cases, non-steroidal antiinflammatory agents were effective, and no additional treatment was needed. Two cases, in which pericardiocentesis was applied to improve the cardiac tamponade, have been reported $[1,2]$. The pericardial effusion in these cases was hemorrhagic, derived from cardiac rupture, and no studies on cardiac tamponade complicated with TC-related inflammatory effusion have been reported. In the present case, the pericardial effusion increased rapidly, resulting in cardiac tamponade regardless of the use of anti-inflammatory agents, including non-steroidal anti-inflammatory agents and colchicine. The effusion sample was exudative and nonhemorrhagic, indicating pericardial inflammation. After the pericardiocentesis, no additional effusion pooled, suggesting improvement in the pericardial inflammation.

As described above, TC-related symptomatic pericarditis is uncommon; however, in the present case and a previous report, pericarditis developed during the TC recovery phase [4]; thus, if a detailed examination is delayed, it is difficult to differentiate pericarditis related to TC from that from other origins. Cases of pericarditis with an identified etiology include malignant diseases (5-10\%) systematic inflammatory disease and pericardial injury syndrome (5-10\%), tuberculosis (4\%), and purulent pericarditis $(<1 \%)$. About $80-90 \%$ of cases are labeled idiopathic, and most are presumed to be viral [9]. Some TC-related cardiomyopathy might be concealed in these cases of idiopathic pericarditis.

The present case had some limitations. First, although pericarditis was indicated, no systemic inflammation was observed. This can be explained by the results of a previous study, which demonstrated fewer systemic inflammation signs in patients with pericarditis with myocardial involvement than in those in simple pericarditis [10]. Second, some reports have suggested specific signs in TC evaluated by CMRI (cardiac edema evaluated by $\mathrm{T} 2$ weighted image) [7], but CMRI used in the present case revealed no remarkable signs. The appearance of signs with this technique is likely to be affected by the timing of the measurement. Myocardial edema has been observed in TC during the subacute phase, but not during the acute or chronic phases [11]. In the 
a)

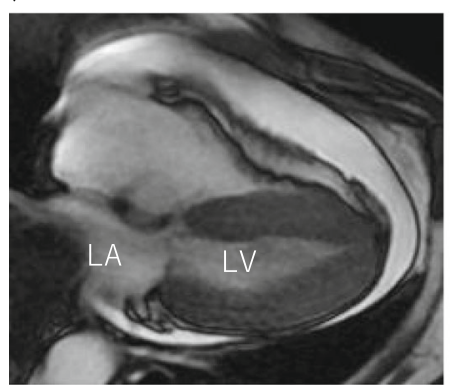

b)

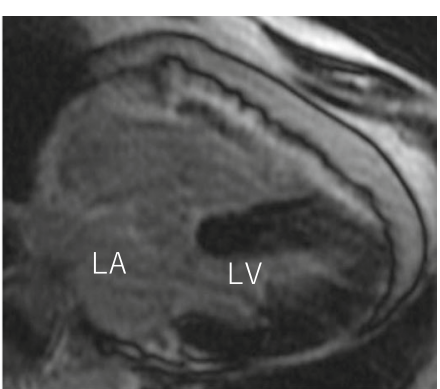

Fig. 7 Magnetic resonance imaging (a: Fat suppressed T2 weighted image b: Delayed gadolinium enhancement image). Neither transmural late gadolinium enhancement nor cardiac edema were observed. (LA, Left Atrium; LV, Left Ventricle)

present case, CMRI was performed 30 days after admission. We might have detected the myocardium edema if we had performed CMRI at the subacute phase. In conclusions, most TC-related pericardial effusion is asymptomatic; however, it is capable of affecting hemodynamics. The condition of these patients should be carefully evaluated, even after improvement of left ventricular function.

\section{Abbreviations \\ CMRI: Cardiac magnetic resonance imaging; DGE: Delayed gadolinium enhancement; ECG: Electrocardiogram; TC: Takotsubo cardiomyopathy}

\section{Acknowledgements}

Not applicable.

\section{Authors' contributions}

$\mathrm{TH}, \mathrm{YN}$, and HM drafted the manuscript. YN wrote the manuscript with support from ST, SU and HF. TK, KS and MK contributed to date and images collection. MT made crucial revision of this article. All authors finally read and approved this article.

\section{Funding}

There was no funding received for this manuscript.

\section{Availability of data and materials}

Not applicable.

\section{Ethics approval and consent to participate}

Written informed consent was obtained from the patient of this case report.

\section{Consent for publication}

Written informed consent was obtained from the patient for publication of this case report and any accompanying images.

\section{Competing interests}

The authors declare that they have no competing interests.

Received: 3 June 2019 Accepted: 3 February 2020

Published online: 06 February 2020

\section{References}

1. Yeh RW, Yu PB, Drachman DE. Takotsubo cardiomyopathy complicated by cardiac tamponade: classic hemodynamic findings with a new disease. Circulation. 2010;122:1239-41. https://doi.org/10.1161/CIRCULATIONAHA.110. 955633

2. ter Bals E, Odekerken DAM, Somsen GA. Takotsubo cardiomyopathy complicated by cardiac tamponade. Netherlands Hear J. 2014;22:246-8. https://doi.org/10.1007/s12471-013-0458-z.
3. Yalta K, Yilmaztepe M, Zorkun C. Left Ventricular Dysfunction in the Setting of Takotsubo Cardiomyopathy: A Review of Clinical Patterns and Practical Implications. Card Fail Rev. 2018;4:14-20. https://doi.org/10.15420/cfr.2018: 24:2.

4. Maruyama T, Hanaoka T, Nakajima H. Acute pericarditis in the recovery phase of transient left ventricular apical ballooning syndrome (takotsubo cardiomyopathy). Intern Med. 2007;46:1857-60. https://doi.org/10.2169/ internalmedicine.46.0184.

5. Burgdorf $\mathrm{C}$, Bonnemeier $\mathrm{H}$. Coronary artery vasospasm or true transient left ventricular apical ballooning? Differentiation by nuclear imaging. J Nucl Cardiol. 2008;15:599-603.

6. María A, Rivera C, Ruiz-bailén M, Aguilar LR. Takotsubo cardiomyopathy - a clinical review. Medical science monitor. 2011:17:135-47.

7. Ingo E, Friedrich MG. Clinical Characteristics and Cardiovascular Magnetic Resonance Findings in Stress (Takotsubo) Cardiomyopathy. JAMA. 2011;306: 277-86

8. Kim J, Laird-Fick HS, Alsara O, Gourineni V, Abela GS. Pericarditis in takotsubo cardiomyopathy: a case report and review of the literature. Case Rep Cardiol. 2013;2013:917851. https://doi.org/10.1155/2013/917851.

9. Imazio M, Gaita F, LeWinter M. Evaluation and treatment of pericarditis: a systematic review. JAMA. 2015;314:1498-506. https://doi.org/10.1001/jama. 2015.12763 .

10. Imazio M, Brucato A, Barbieri A, Ferroni F, Maestroni S, Ligabue G, et al. Good prognosis for pericarditis with and without myocardial involvement: results from a multicenter, prospective cohort study. Circulation. 2013;128: 42-9. https://doi.org/10.1161/CIRCULATIONAHA.113.001531.

11. Kato K, Daimon M, Ishibashi I, Kobayashi Y. Myocardial edema in Takotsubo syndrome - serial cardiovascular magnetic resonance imaging of the natural course. Circ J. 2017:81:1368-9. https://doi.org/10.1253/circj.cj-170065

\section{Publisher's Note}

Springer Nature remains neutral with regard to jurisdictional claims in published maps and institutional affiliations.

Ready to submit your research? Choose BMC and benefit from:

- fast, convenient online submission

- thorough peer review by experienced researchers in your field

- rapid publication on acceptance

- support for research data, including large and complex data types

- gold Open Access which fosters wider collaboration and increased citations

- maximum visibility for your research: over $100 \mathrm{M}$ website views per year

At BMC, research is always in progress.

Learn more biomedcentral.com/submissions 